\title{
TWO-DIMENSIONAL RUIN PROBABILITY FOR SUBEXPONENTIAL CLAIM SIZE*
}

\author{
BY
}

SERGEY F OSS (EDINBURgh AND NOVOSIbIRSK), DMITRY KORSHUNOV (LANCASTER), ZBIGNIEW PALMOWS K I (WrocŁaW), AND TOMASZ ROLSK I (WROCŁAW)

\begin{abstract}
We analyse the asymptotics of ruin probabilities of two insurance companies (or two branches of the same company) that divide between them both claims and premiums in some specified proportions when the initial reserves of both companies tend to infinity, and generic claim size is subexponential.
\end{abstract}

2010 AMS Mathematics Subject Classification: Primary: 60G51, 60G50; Secondary: 60K25.

Key words and phrases: Two-dimensional risk process, ruin probability, subexponential distribution.

\section{INTRODUCTION}

We consider a model of two-dimensional risk process with renewal input: claims $\vec{\sigma}_{n}=\left(\sigma_{n, 1}, \sigma_{n, 2}\right)$ arrive in a random input at arrival epochs $\left\{t_{n}\right\}$ with interarrival times $\left\{\tau_{n}\right\}$. There are two insurance companies, company $i$ has initial capital $x_{i}$ and premium rate $p_{i}$, and covers claims $\sigma_{n, i}, i=1,2$. We assume that the two sequences $\left\{\vec{\sigma}_{n}\right\}$ and $\left\{\tau_{n}\right\}$ are mutually independent and that each of them consists of i.i.d. random variables.

Let $N(t)$ be the number of claims by time $t \geqslant 0$,

$$
N(t)=\max \left\{n: t_{n} \leqslant t\right\} ;
$$

then

$$
\vec{S}(t)=\left(S_{1}(t), S_{2}(t)\right):=\sum_{n=1}^{N(t)} \vec{\sigma}_{n}
$$

* This work is partially supported by the National Science Centre under the grants 2013/09/B/HS4/01496 and 2015/17/B/ST1/01102 (2016-2019). The work of the first co-author was supported by RSF research grant no. 17-11-01173. The second and third authors kindly acknowledge partial support by the project RARE-318984, a Marie Curie IRSES Fellowship within the Seventh European Community Framework Programme. 
is the vector of total claim sizes by time $t$. Further, let

$$
\vec{b}(t)=\left(b_{1}(t), b_{2}(t)\right):=\vec{x}+\vec{p} t
$$

be the sum of initial capitals and of total premiums by time $t$, here $\vec{x}=\left(x_{1}, x_{2}\right)$ and $\vec{p}=\left(p_{1}, p_{2}\right)$. Introduce ruin probabilities of two types: for any $T \in(0, \infty]$, these are

$$
\begin{aligned}
& \psi_{\wedge}(\vec{x}, T)=\mathbb{P}\left\{S_{1}(t)>b_{1}(t) \text { or } S_{2}(t)>b_{2}(t) \text { for some } t \leqslant T\right\}, \\
& \psi_{\vee}(\vec{x}, T)=\mathbb{P}\left\{S_{1}(t)>b_{1}(t) \text { and } S_{2}(t)>b_{2}(t) \text { for some } t \leqslant T\right\} .
\end{aligned}
$$

Here $\psi_{\wedge}\left(x_{1}, x_{2}, T\right)$ describes the ruin probability of at least one insurance company, while $\psi_{\vee}\left(x_{1}, x_{2}, T\right)$ corresponds to the ruin of the both insurance companies.

There are several papers - see, e.g., Konstantinides and $\mathrm{Li}$ [16] and the list of references therein - where it is assumed that the pairs $\left(\sigma_{n, 1}, \sigma_{n, 2}\right)$ are i.i.d. and have a multivariate regularly varying distribution. We like to consider claim sizes with distributions from a more general subexponential class that includes Pareto and also log-normal and Weibull distributions. We are unaware of any reasonable concept of subexponentiality here. So only two extreme options seem to be doable: either (i) $\sigma_{n, 1}$ and $\sigma_{n, 2}$ are independent or (ii) they are dependent deterministically, say

$$
\sigma_{n, 1}=l\left(\sigma_{n}\right) \quad \text { and } \quad \sigma_{n, 1}=\sigma_{n}-l\left(\sigma_{n}\right),
$$

where $0 \leqslant l(x) \leqslant x$ and $\sigma_{n}$ is the cumulative claim of the client $n$ that is covered by the two insurance companies together.

There are several papers that study related problems in direction (i) - see, e.g., Li et al. [18], Chen et al. [8], Chen, Wang, Wang [6], [7], Wang et al. [22], Jiang et al. [15], Hu and Jiang [114], Lu and Zhang [20], see also references therein. In particular, in the paper by Lu and Zhang [20], a uniform asymptotics over finite intervals has been obtained under some restrictive assumptions on distributions.

Avram et al. [3], [4] studied boundary crossing probabilities of a stochastic process with light-tailed increments. This study was also motivated by ruin probabilities of two insurance companies with proportional claims and the steady state distribution of a tandem queue with two servers (see [19]). Similar considerations were done in Badila et al. [5] (for mutidimensional risk process) and in [14] (for the two-dimensional risk process with constant interest rate).

The key message of this paper is that in both cases results on the uniform asymptotics may be obtained for a general class of strong subexponential distributions, no further restrictions are needed. We only consider the second direction using approach developed in a series of works of Denisov, Foss, Korshunov, Palmowski, and Zachary (see, e.g., [9], [11]]-[133], [17]); similar arguments apply in the first direction.

To make it simple, we assume $l(x)$ to be linear, $l(x)=l x$ for some $l \in(0,1)$. Then, after some transformations we can reformulate the problem as follows. Let 
us define

$$
S_{t}=\sum_{i=1}^{N_{t}} \sigma_{i}
$$

where $N_{t}$ is a renewal process with positive i.i.d. interarrival times $\tau_{i}$, and the claim sizes $\sigma_{i}$ are positive i.i.d. random variables that do not depend on $N(t)$ and have a common distribution function $F(x)$. Note also that $N_{t}=\max \{k \geqslant 0$ : $\left.t_{k} \leqslant t\right\}$ for a random walk constructed from the interarrival times:

$$
t_{n}=\sum_{k=1}^{n} \tau_{k}
$$

Let the boundaries $b_{1}$ and $b_{2}$ be given by

$$
b_{1}(t)=b_{1}\left(t ; x_{1}\right)=x_{1}+p_{1} t, \quad b_{2}(t)=b_{2}\left(t ; x_{2}\right)=x_{2}+p_{2} t .
$$

We assume that $\mathbb{E} \sigma<\infty, \mathbb{E} \tau<\infty$ and that

$$
p_{1}>p_{2}, \quad p_{2}>\rho:=\mathbb{E}[\sigma] / \mathbb{E}[\tau]
$$

for generic $\tau$ and $\sigma$. In this paper, for $T>0$, we will consider the boundary crossing probabilities

$$
\begin{aligned}
& \psi_{\wedge}\left(x_{1}, x_{2}, T\right)=\mathbb{P}\left\{\tau_{\wedge}\left(x_{1}, x_{2}\right) \leqslant T\right\} \\
& \psi_{\vee}\left(x_{1}, x_{2}, T\right)=\mathbb{P}\left\{\tau_{\vee}\left(x_{1}, x_{2}\right) \leqslant T\right\}
\end{aligned}
$$

for

$$
\begin{aligned}
& \tau_{\wedge}\left(x_{1}, x_{2}\right)=\inf \left\{t \geqslant 0: S_{t}>b_{1}(t) \wedge b_{2}(t)\right\}, \\
& \tau_{\vee}\left(x_{1}, x_{2}\right)=\inf \left\{t \geqslant 0: S_{t}>b_{1}(t) \vee b_{2}(t)\right\},
\end{aligned}
$$

where $x \wedge y=\min \{x, y\}$ and $x \vee y=\max \{x, y\}$.

The $\psi_{\wedge}\left(x_{1}, x_{2}, T\right)$ describes the ruin probability of at least one insurance company before time $T$; if $T=\infty$, then

$$
\psi_{\wedge}\left(x_{1}, x_{2}\right):=\psi_{\wedge}\left(x_{1}, x_{2}, \infty\right)
$$

is a ruin probability of at least one company. The $\psi_{\vee}\left(x_{1}, x_{2}, T\right)$ and

$$
\psi_{\vee}\left(x_{1}, x_{2}\right):=\psi_{\vee}\left(x_{1}, x_{2}, \infty\right)
$$

correspond to the ruin of both insurance companies. The first assumption in ([L.2) means that the second company has a smaller premium rate than the first company, and the second assumption is the condition under which reserves of both insurance 
companies tend to infinity. The solutions to the "degenerate two-dimensional" ruin problems strongly depend on the relative position of the vector of premium rates $p=\left(p_{1}, p_{2}\right)$ with respect to the proportions vector $(1,1)$. Namely, if the initial reserves satisfy $x_{2} \leqslant x_{1}$, the two lines do not intersect. It follows therefore that $\psi_{\wedge}$ and $\psi_{\vee}$ are ruin probabilities of the second and first companies, respectively. In this case the asymptotics follows from one-dimensional ruin theory, see, e.g., Rolski et al. [21]]. Therefore, we focus here on the opposite case where

$$
x_{1}<x_{2}
$$

In this paper we derive the exact first order asymptotics of these ruin probabilities if $x_{1}, x_{2}$ tend to infinity and when the claims follow a subexponential distribution. We model the claims by subexponential distributions since many catastrophic events like earthquakes, storms, terrorist attacks, etc. are used in their description. Insurance companies use, e.g., the log-normal distribution (which is subexponential) to model car claims - see Foss et al. [1]], Rolski et al. [2]] or Embrechts et al. [10] for the further background.

The paper is organized as follows. In the next section we present the main results which will be proved in Section 3 .

\section{MAIN RESULTS}

In order to state our results we recall basic notation and notions. Hereinafter we write $f(x, y) \sim g(x, y)$ if $f(x, y) / g(x, y) \rightarrow 1$ as $x, y \rightarrow \infty$ and $f(x) \sim g(x)$ if $f(x) / g(x) \rightarrow 1$ as $x \rightarrow \infty$. For a distribution $F, \bar{F}$ denotes the tail distribution function given by $\bar{F}(x)=1-F(x)$.

A distribution $F$ on $\mathbb{R}^{+}$is called subexponential $(F \in \mathcal{S})$ if and only if $\bar{F}(x)>0$ for all $x$ and

$$
\overline{F^{* 2}}(x) \sim 2 \bar{F}(x) \text { as } x \rightarrow \infty,
$$

where $F^{* 2}$ is the convolution of $F$ with itself.

A distribution $F$ on $\mathbb{R}^{+}$is called strong subexponential $\left(F \in \mathcal{S}^{*}\right)$ if and only if $\bar{F}(x)>0$ for all $x$ and

$$
\int_{0}^{x} \bar{F}(x-y) \bar{F}(y) d y \sim 2 m_{F} \bar{F}(x) \quad \text { as } x \rightarrow \infty
$$

where

$$
m_{F}=\int_{0}^{\infty} \bar{F}(x) d x
$$

is the expectation of $F$. It is known that the property $F \in \mathcal{S}^{*}$ is a tail property of $F$, namely, if $F_{1} \in \mathcal{S}^{*}$ and $\bar{F}_{1}(x) \sim \bar{F}_{2}(x)$ as $x \rightarrow \infty$, then $F_{2} \in \mathcal{S}^{*}$. Further, if 
$F \in \mathcal{S}^{*}$, then $F \in \mathcal{S}$ and also $F^{s} \in \mathcal{S}$, where

$$
\overline{F^{s}}(x)=\min \left(1, \int_{x}^{\infty} \bar{F}(t) d t\right)
$$

is the integrated tail distribution function determined by $F$; see [I]] for details.

Let us put

$$
m_{i}=p_{i} \mathbb{E} \tau-\mathbb{E} \sigma, \quad i=1,2 .
$$

Since $p_{1}>p_{2}$, we have $m_{1}>m_{2}$.

THEOREM 2.1. Assume that the distribution $F$ of generic $\sigma \geqslant 0$ is strong subexponential. Then, as $x_{1}, x_{2} \rightarrow \infty$,

$$
\psi_{\wedge}\left(x_{1}, x_{2}, T\right) \sim H_{T}\left(x_{1}, x_{2}\right):=\int_{0}^{\mathbb{E} N_{T}} \bar{F}\left(\min \left\{x_{1}+t m_{1}, x_{2}+t m_{2}\right\}\right) d t
$$

and

$$
\psi_{\vee}\left(x_{1}, x_{2}, T\right) \sim U_{T}\left(x_{1}, x_{2}\right):=\int_{0}^{\mathbb{E} N_{T}} \bar{F}\left(\max \left\{x_{1}+t m_{1}, x_{2}+t m_{2}\right\}\right) d t
$$

hold uniformly for all $T>0$.

COROLlARY 2.1. In conditions of Theorem 2.1 , as $x_{1}, x_{2}$ and $T \rightarrow \infty$,

$$
\psi_{\wedge}\left(x_{1}, x_{2}, T\right) \sim \int_{0}^{T / \mathbb{E} \tau} \bar{F}\left(\min \left\{x_{1}+t m_{1}, x_{2}+t m_{2}\right\}\right) d t
$$

and

$$
\psi_{\vee}\left(x_{1}, x_{2}, T\right) \sim \int_{0}^{T / \mathbb{E} \tau} \bar{F}\left(\max \left\{x_{1}+t m_{1}, x_{2}+t m_{2}\right\}\right) d t .
$$

COROLlARY 2.2. Assume that $F$ is strong subexponential and $a<1$ is fixed. Then we have the following asymptotics as $x \rightarrow \infty$ :

$$
\psi_{\wedge}(a x, x) \sim H(x):=\int_{0}^{\infty} \bar{F}\left(\min \left\{a x+t m_{1}, x+t m_{2}\right\}\right) d t
$$

and

$$
\psi_{\vee}(a x, x) \sim U(x):=\int_{0}^{\infty} \bar{F}\left(\max \left\{a x+t m_{1}, x+t m_{2}\right\}\right) d t .
$$


We can also identify the joint distribution of the ruin times ([L.3) and (ㄴ.4) and the position at the moment at these ruin times. To do this we will require additional assumptions. Let $e(x)$ be a function tending to infinity as $x \rightarrow \infty$. For fixed $y \geqslant 0$ and $v \geqslant 0$ we write

$$
H^{y, v}(x)=\int_{y e(x)}^{\infty} \bar{F}\left(\min \left\{a x+t m_{1}, x+t m_{2}\right\}+v e(x)\right) d t
$$

and

$$
U^{y, v}(x)=\int_{y e(x)}^{\infty} \bar{F}\left(\max \left\{a x+t m_{1}, x+t m_{2}\right\}+v e(x)\right) d t .
$$

Note that $H(x)=H^{0,0}(x)$ and $U(x)=U^{0,0}(x)$. We will assume that there exists some continuous distribution function $G$ such that

$$
\lim _{x \rightarrow \infty} \frac{H^{y, v}(x)}{H(x)}=\bar{G}\left(m_{1} y+v\right)
$$

and that

$$
\lim _{x \rightarrow \infty} \frac{U^{y, v}(x)}{U(x)}=\bar{G}\left(m_{2} y+v\right) .
$$

THEOREM 2.2. Assume that there exist a function $e(x) \uparrow \infty$ and a continuous probability distribution $G$ on the positive half-line $(0, \infty)$ such that (2.12) and (2.13) are satisfied. Then, for $a<1$, we have

$\lim _{x \rightarrow \infty} \mathbb{P}\left\{\frac{\tau_{\wedge}(a x, x)}{e(x)} \geqslant y, \frac{S_{\tau_{\wedge}(a x, x)}-b_{\wedge}(t)}{e(x)} \geqslant v \mid \tau_{\wedge}(a x, x)<\infty\right\}=\bar{G}\left(m_{1} y+v\right)$

and

$\lim _{x \rightarrow \infty} \mathbb{P}\left\{\frac{\tau_{\vee}(a x, x)}{e(x)} \geqslant y, \frac{S_{\tau_{\vee}(a x, x)}-b_{\vee}(t)}{e(x)} \geqslant v \mid \tau_{\vee}(a x, x)<\infty\right\}=\bar{G}\left(m_{2} y+v\right)$.

For similar statements for a linear boundary, see Asmussen and Klüppelberg [2] and Foss et al. [II] in the case of i.i.d. jumps, and Asmussen and Foss [I] in the case of Markov modulation.

REMARK 2.1. To provide examples where assumptions ([2.12) and ([2.13) are satisfied, we choose strong subexponential distribution $F$ for which $F^{s}$ is selfneglecting. That is, let us assume that there exist a function $e(x) \uparrow \infty$ and a continuous probability distribution $G$ on the positive half-line $(0, \infty)$ such that, for any $y>0$,

$$
\frac{\bar{F}^{s}(x+y e(x))}{\bar{F}^{s}(x)} \rightarrow \bar{G}(y) \quad \text { as } x \rightarrow \infty .
$$


From [110], Theorem 3.4.5, p. 158, it follows that $F^{s}$ is either in the domain of attraction of Fréchet distribution or in the domain of attraction of Gumbel distribution. In the first case,

$$
F \in \operatorname{RV}(\alpha+1), \quad F^{s} \in \operatorname{RV}(\alpha), \quad \bar{G}(y)=\left(1+\alpha^{-1} y\right)^{-\alpha}, \quad e(x)=\alpha^{-1} x,
$$

with $\alpha>0$ (then $\mathbb{E} \sigma<\infty$ ) for the family $\mathrm{RV}(\alpha+1)$ of regularly varying distributions. In the second case,

$$
\bar{G}(y)=e^{-y}, \quad e(x)=\frac{m_{F} \bar{F}^{s}(x)}{\bar{F}(x)} .
$$

To check (2.12) it is convenient to use the representation

$$
\begin{aligned}
H^{y, v}(x)= & \frac{1}{m_{1}} \bar{F}^{s}\left(a x+\left(m_{1} y+v\right) e(x)\right) \\
& +\left(\frac{1}{m_{2}}-\frac{1}{m_{1}}\right) \bar{F}^{s}\left(a x+m_{1} \widehat{T}+v e(x)\right) \\
& +\frac{1}{m_{2}} \bar{F}^{s}\left(x+m_{2} \widehat{T}+v e(x)\right)
\end{aligned}
$$

for the moment when two lines $a x+m_{1} t$ and $x+m_{2} t$ cut each other:

$$
\widehat{T}=\frac{(1-a) x}{m_{1}-m_{2}} .
$$

Recall also that $H(x)=H^{0,0}(x)$. Now one can check that if (2.17) holds true, then indeed (2.12) is satisfied. Similarly, one can prove that in this case the assumption (2.12) is also satisfied.

The case (2.18) is much more advanced and it should be analysed case by case using, for example, [110], Example 3.3.35, p. 149.

In our proof of Theorem 2.1 , we use the following simple result that may be of use in other settings.

Lemma 2.1. Let $z(t) \geqslant 0$ be an increasing function, $F$ a distribution, and $N_{t}$ a renewal process. Then

$$
\mathbb{E} \int_{0}^{N_{T}} \bar{F}(x+z(t)) d t \leqslant \int_{0}^{\mathbb{E} N_{T}} \bar{F}(x+z(t)) d t \quad \text { for all } x \text { and } T>0
$$

and

$$
\mathbb{E} \int_{0}^{N_{T}} \bar{F}(x+z(t)) d t \sim \int_{0}^{\mathbb{E} N_{T}} \bar{F}(x+z(t)) d t \quad \text { as } x, T \rightarrow \infty .
$$


If, in addition, the distribution $F$ is long-tailed, then

(2.22)

$\mathbb{E} \int_{0}^{N_{T}} \bar{F}(x+z(t)) d t \sim \int_{0}^{\mathbb{E} N_{T}} \bar{F}(x+z(t)) d t \quad$ as $x \rightarrow \infty$ uniformly for all $T>0$.

Notice that, in general, the last result is not applicable to an upper limit $N_{\varrho}$, where $\varrho$ is a stopping time like in (3.16) and (3.17) below.

\section{PROOFS}

3.1. Proof of Theorem 2.1. As discussed above, it is sufficient to consider the case $x_{2}>x_{1}$. For any $x>0$ and $\widehat{T}>0$, let $\widehat{x}=x+p_{1} \widehat{T}$ and

$$
\widehat{b}(t):= \begin{cases}x+p_{1} t & \text { for } t \leqslant \widehat{T}, \\ \widehat{x}+p_{2}(t-\widehat{T}) & \text { for } t>\widehat{T}\end{cases}
$$

which is a continuous piecewise linear function. Then both assertions of the theorem, (2.4) and (2.5), will hold if the following tail asymptotics is proven for all $p_{1}>0$ and $p_{2}>0$, without assumption $p_{2}<p_{1}$ :

$$
\begin{aligned}
\psi(x, \widehat{T}, T) & :=\mathbb{P}\left\{S_{t}>\widehat{b}(t) \text { for some } t \leqslant T\right\} \\
& \sim \int_{0}^{\mathbb{E} N_{T}} \bar{F}(\widehat{x}(t)) d t \quad \text { as } x \rightarrow \infty \text { uniformly for all } \widehat{T} \text { and } T,
\end{aligned}
$$

where

$$
\widehat{x}(t):= \begin{cases}x+m_{1} t & \text { for } t \leqslant \widehat{T} / \mathbb{E} \tau, \\ x+m_{1} \widehat{T} / \mathbb{E} \tau+m_{2}(t-\widehat{T} / \mathbb{E} \tau) & \text { for } t>\widehat{T} / \mathbb{E} \tau .\end{cases}
$$

Since $F \in \mathcal{S}^{*}, F$ is particularly subexponential and long-tailed. For any fixed $T$, the random variable $N_{T}$ has a light tail, that is, it has a finite exponential moment. In addition, $N_{T}$ is independent of $\sigma$ 's. Hence, by Theorem 3.37 in [I]], for every fixed $T$,

$$
\mathbb{P}\left\{\sum_{i: t_{i} \leqslant T} \sigma_{i}>x\right\}=\sum_{j=0}^{\infty} \mathbb{P}\left\{N_{T}=j\right\} \overline{F^{* j}}(x) \sim \mathbb{E} N_{T} \bar{F}(x) \quad \text { as } x \rightarrow \infty,
$$

and this equivalence holds uniformly on any $T$-compact set. This observation together with the lower and upper bounds

$$
\mathbb{P}\left\{\sum_{i: t_{i} \leqslant T} \sigma_{i}>x+\max \left\{p_{1}, p_{2}\right\} T\right\} \leqslant \psi(x, \widehat{T}, T) \leqslant \mathbb{P}\left\{\sum_{i: t_{i} \leqslant T} \sigma_{i}>x\right\}
$$


and long-tailedness of $F$ implies that, uniformly on any $T$-compact set,

$$
\psi(x, \widehat{T}, T) \sim \mathbb{E} N_{T} \bar{F}(x) \quad \text { as } x \rightarrow \infty .
$$

Taking into account that

$$
N_{T} \bar{F}\left(x+\max \left\{m_{1}, m_{2}\right\} T\right) \leqslant \int_{0}^{N_{T}} \bar{F}(\widehat{x}(t)) d t \leqslant N_{T} \bar{F}(x)
$$

and that $F$ is long-tailed, we prove that (B.D) holds as $x \rightarrow \infty$ uniformly on $T$ compact sets.

Therefore, it remains to prove (B.T) for the case $T \rightarrow \infty$. If $T \leqslant \widehat{T}$, then

$$
\psi(x, \widehat{T}, T)=\mathbb{P}\left\{\sum_{j=1}^{n} \sigma_{j}>x+p_{1} t_{n} \text { for some } n \leqslant N_{T}\right\},
$$

so, as proven in [17], Theorem 3, for the supremum of a compound renewal process with negative drift $\mathbb{E} \sigma / \mathbb{E} \tau-p_{1}=-m_{1} / \mathbb{E} \tau$, as $x \rightarrow \infty$ uniformly for $T \leqslant \widehat{T}$,

$$
\begin{aligned}
\psi(x, \widehat{T}, T) & \sim \frac{1}{m_{1}} \int_{0}^{m_{1} \mathbb{E} N_{T}} \bar{F}(x+t) d t \\
& =\int_{0}^{\mathbb{E} N_{T}} \bar{F}\left(x+m_{1} t\right) d t \sim \int_{0}^{\mathbb{E} N_{T}} \bar{F}(\widehat{x}(t)) d t,
\end{aligned}
$$

where the last equivalence follows because $\mathbb{E} N_{T} \sim T / \mathbb{E} \tau$ as $T \rightarrow \infty$ and, for any $\varepsilon>0$,

$$
\begin{aligned}
& (1-\varepsilon) \int_{0}^{T / \mathbb{E} \tau} \bar{F}\left(x+m_{1} t\right) d t \leqslant \int_{0}^{(1-\varepsilon) T / \mathbb{E} \tau} \bar{F}\left(x+m_{1} t\right) d t \\
& \quad \leqslant \int_{0}^{(1+\varepsilon) T / \mathbb{E} \tau} \bar{F}\left(x+m_{1} t\right) d t \leqslant(1+\varepsilon) \int_{0}^{T / \mathbb{E} \tau} \bar{F}\left(x+m_{1} t\right) d t
\end{aligned}
$$

and similar bounds for the integral of $\bar{F}(\widehat{x}(t))$.

Let us now consider the case $T \rightarrow \infty$ and $T>\widehat{T}$. If $\widehat{T}$ is bounded, then we make use of the inequalities

$$
\begin{aligned}
& \mathbb{P}\left\{S_{t}>x+p_{1} \widehat{T}+p_{2} t \text { for some } t \leqslant T\right\} \leqslant \psi(x, \widehat{T}, T) \\
& \quad \leqslant \mathbb{P}\left\{S_{t}>x-p_{1} \widehat{T}+p_{2} t \text { for some } t \leqslant T\right\},
\end{aligned}
$$

which reduce - due to long-tailedness of $F-$ the problem to the case $\widehat{T}=T$, a particular case of that considered above. 
The case where $T \rightarrow \infty, \widehat{T} \rightarrow \infty$ and $T>\widehat{T}$, but $T-\widehat{T}$ is bounded, is very similar. Indeed, in this case it is enough to notice that

$$
\begin{aligned}
\mathbb{P}\left\{S_{t}>x+p_{2}(T-\widehat{T})+p_{1} t \text { for some } t \leqslant T\right\} \leqslant \psi(x, \widehat{T}, T) \\
\leqslant \mathbb{P}\left\{S_{t}>x-p_{2}(T-\widehat{T})+p_{1} t \text { for some } t \leqslant T\right\} .
\end{aligned}
$$

Let us now consider the last remaining case where $T \rightarrow \infty, \widehat{T} \rightarrow \infty$, and $T-\widehat{T} \rightarrow \infty$. We demonstrate two approaches, where the first one is based on the uniform equivalences in the case of linear functions obtained in [17] and the second on the discrete time results from [12].

3.2. Proof based on [17]. Since $F$ is particularly long-tailed, there exists an increasing function $h(x) \rightarrow \infty$ such that $h(x)=o(x)$ and

$$
\bar{F}(x+h(x)) \sim \bar{F}(x) \text { as } x \rightarrow \infty,
$$

see [11]], Lemma 2.19. For $T>\widehat{T}$,

$$
\begin{aligned}
\psi(x, \widehat{T}, T) \leqslant & \mathbb{P}\left\{\sum_{j=1}^{n} \sigma_{j}>x+p_{1} t_{n}-h(x) \text { for some } n \leqslant N_{\widehat{T}}\right\} \\
& +\mathbb{P}\left\{\sum_{j=1}^{n} \sigma_{j} \leqslant x+p_{1} t_{n}-h(x) \text { for all } n \leqslant N_{\widehat{T}},\right. \\
\left.\qquad \sum_{j=1}^{n} \sigma_{j}>\widehat{x}\left(t_{n}\right) \text { for some } n \in\left(N_{\widehat{T}}, N_{T}\right]\right\} & \\
= & P_{1}+P_{2} .
\end{aligned}
$$

Again by [117], Theorem 3,

$$
P_{1} \sim \int_{0}^{\mathbb{E} N_{\widehat{T}}} \bar{F}\left(x-h(x)+m_{1} t\right) d t \sim \int_{0}^{\mathbb{E} N_{\widehat{T}}} \bar{F}\left(x+m_{1} t\right) d t \quad \text { as } x \rightarrow \infty,
$$

as follows from (B.6).

The second probability on the right-hand side of (B.7) equals

$$
\begin{aligned}
& P_{2}=\mathbb{P}\left\{S_{\widehat{T}} \leqslant x+p_{1} \widehat{T}-h(x),\right. \\
& \left.S_{\widehat{T}}-p_{1} \widehat{T}+\sum_{j=N_{\widehat{T}}+1}^{n} \sigma_{j}-p_{2}\left(t_{n}-\widehat{T}\right)>x \text { for some } n \in\left[N_{\widehat{T}}+1, N_{T}\right]\right\} .
\end{aligned}
$$

We need to exclude dependence caused by the interval $\left(\widehat{T}, t_{N_{\widehat{T}}+1}\right]$. We do it in the following way:

$$
\begin{aligned}
\sup _{n \in\left[N_{\widehat{T}}+1, N_{T}\right]}( & \left.\sum_{j=N_{\widehat{T}}+1}^{n} \sigma_{j}-p_{2}\left(t_{n}-\widehat{T}\right)\right) \\
& \leqslant \sigma_{N_{\widehat{T}}+1}+\sup _{n \in\left[N_{\widehat{T}}+2, N_{T}\right]}\left(\sum_{j=N_{\widehat{T}}+2}^{n} \sigma_{j}-p_{2}\left(t_{n}-t_{N_{\widehat{T}}+1}\right)\right),
\end{aligned}
$$


where the two random variables on the right-hand side are independent because $t_{N_{\widehat{T}}+1}$ is a stopping time. Further, extending the interval $\left(t_{N_{\widehat{T}}+1}, T\right]$ to the interval $\left(t_{N_{\widehat{T}}+1}, t_{N_{\widehat{T}}+1}+T-\widehat{T}\right]$ of length $T-\widehat{T}$, we conclude that

$$
\sup _{n \in\left[N_{\widehat{T}}+2, N_{T}\right]}\left(\sum_{j=N_{\widehat{T}}+2}^{n} \sigma_{j}-p_{2}\left(t_{n}-t_{N_{\widehat{T}}+1}\right)\right)
$$

is stochastically not greater than

$$
\zeta_{T-\widehat{T}}:=\sup _{t \leqslant T-\widehat{T}}\left(S_{t}-p_{2} t\right),
$$

whose tail is equivalent to, again by [17], Theorem 3,

$$
\mathbb{P}\{\zeta>z\} \sim \int_{0}^{\mathbb{E} N_{T-\widehat{T}}} \bar{F}\left(z+m_{2} y\right) d y \quad \text { as } z \rightarrow \infty .
$$

Fix any $\varepsilon>0$ such that $m_{1}=p_{1} \mathbb{E} \tau-\mathbb{E} \sigma>\varepsilon$. Then it follows from (B.9) that, with $\widetilde{\zeta}_{T-\widehat{T}}$ being an independent copy of $\zeta_{T-\widehat{T}}$,

$$
\begin{aligned}
P_{2} \leqslant & \int_{-\infty}^{x-h(x)} \mathbb{P}\left\{S_{\widehat{T}}-p_{1} \widehat{T} \in d y\right\} \mathbb{P}\left\{\sigma_{N_{\widehat{T}}+1}+\widetilde{\zeta}_{T-\widehat{T}}>x-y\right\} \\
= & \int_{-\infty}^{x-h(x)} \mathbb{P}\left\{S_{\widehat{T}}-p_{1} \widehat{T}+\left(m_{1}-\varepsilon\right) \widehat{T} / \mathbb{E} \tau \in d y+\left(m_{1}-\varepsilon\right) \widehat{T} / \mathbb{E} \tau\right\} \\
& \times \mathbb{P}\left\{\sigma_{N_{\widehat{T}}+1}+\widetilde{\zeta}_{T-\widehat{T}}>x-y\right\} \\
= & \int_{-\infty}^{x+\left(m_{1}-\varepsilon\right) \widehat{T} / \mathbb{E} \tau-h(x)} \mathbb{P}\left\{S_{\widehat{T}}-(\mathbb{E} \sigma+\varepsilon) \widehat{T} / \mathbb{E} \tau \in d y\right\} \\
& \times \mathbb{P}\left\{\sigma_{N_{\widehat{T}}+1}+\widetilde{\zeta}_{T-\widehat{T}}>x+\left(m_{1}-\varepsilon\right) \widehat{T} / \mathbb{E} \tau-y\right\} .
\end{aligned}
$$

We have

$$
\mathbb{P}\left\{\sigma_{N_{\widehat{T}}+1}+\widetilde{\zeta}_{T-\widehat{T}}>u\right\} \sim \mathbb{P}\left\{\widetilde{\zeta}_{T-\widehat{T}}>u\right\}+\bar{F}(u) \quad \text { as } u \rightarrow \infty,
$$

by [I]], Corollary 3.16. Since the process $S_{t}-(\mathbb{E} \sigma+\varepsilon) t / \mathbb{E} \tau$ has negative drift $-\varepsilon / \mathbb{E} \tau$ and its value at time $\widehat{T}$ does not exceed the value of its maximum at the same time, we also have

$$
\mathbb{P}\left\{S_{\widehat{T}}-(\mathbb{E} \sigma+\varepsilon) \widehat{T} / \mathbb{E} \tau>u\right\} \leqslant c_{1}(\varepsilon) \mathbb{P}\left\{\zeta_{\widehat{T}}>u\right\},
$$

where

$$
\zeta_{\widehat{T}}:=\sup _{t \leqslant \widehat{T}}\left(S_{t}-p_{1} t\right)
$$


Integrating (B.10) by parts, applying the last upper bound and then integrating by parts back, we deduce that

$$
\begin{aligned}
& \quad \int_{h\left(x_{1}\right)}^{x+\left(m_{1}-\varepsilon\right) \widehat{T} / \mathbb{E} \tau-h(x)} \mathbb{P}\left\{S_{\widehat{T}}-(\mathbb{E} \sigma+\varepsilon) \widehat{T} / \mathbb{E} \tau \in d y\right\} \\
& \quad \times \mathbb{P}\left\{\sigma_{N_{\widehat{T}}+1}+\widetilde{\zeta}_{T-\widehat{T}}>x+\left(m_{1}-\varepsilon\right) \widehat{T} / \mathbb{E} \tau-y\right\} \\
& =o\left(\mathbb{P}\left\{\zeta_{T-\widehat{T}}>x+\left(m_{1}-\varepsilon\right) \widehat{T} / \mathbb{E} \tau\right\}+\mathbb{P}\left\{\zeta_{\widehat{T}}>x+\left(m_{1}-\varepsilon\right) \widehat{T} / \mathbb{E} \tau\right\}\right),
\end{aligned}
$$

by [II]], Theorem 3.28 , because $F \in \mathcal{S}^{*}$. Hence,

$$
\begin{aligned}
P_{2} \leqslant & \int_{-\infty}^{h(x)} \mathbb{P}\left\{S_{\widehat{T}}-(\mathbb{E} \sigma+\varepsilon) \widehat{T} / \mathbb{E} \tau \in d y\right\} \\
& \times \mathbb{P}\left\{\sigma_{N_{\widehat{T}}+1}+\widetilde{\zeta}_{T-\widehat{T}}>x+\left(m_{1}-\varepsilon\right) \widehat{T} / \mathbb{E} \tau-y\right\} \\
& +o\left(\mathbb{P}\left\{\zeta_{T-\widehat{T}}>x+\left(m_{1}-\varepsilon\right) \widehat{T} / \mathbb{E} \tau\right\}+\mathbb{P}\left\{\zeta_{\widehat{T}}>x+\left(m_{1}-\varepsilon\right) \widehat{T} / \mathbb{E} \tau\right\}\right) \\
\leqslant & \mathbb{P}\left\{\sigma_{N_{\widehat{T}}+1}+\widetilde{\zeta}_{T-\widehat{T}}>x+\left(m_{1}-\varepsilon\right) \widehat{T} / \mathbb{E} \tau-h(x)\right\}+o(\ldots) \\
\sim & \mathbb{P}\left\{\widetilde{\zeta}_{T-\widehat{T}}>x+\left(m_{1}-\varepsilon\right) \widehat{T} / \mathbb{E} \tau\right\}+o\left(\mathbb{P}\left\{\zeta_{\widehat{T}}>x+\left(m_{1}-\varepsilon\right) \widehat{T} / \mathbb{E} \tau\right\}\right) \\
& \quad \mathbb{E} N_{T}-\mathbb{E} N_{\widehat{T}} \bar{F}\left(x+\left(m_{1}-\varepsilon\right) \widehat{T} / \mathbb{E} \tau+m_{2} t\right) d t \\
& \quad \int_{0} o\left(\mathbb{P}\left\{\zeta_{\widehat{T}}>x+\left(m_{1}-\varepsilon\right) \widehat{T} / \mathbb{E} \tau\right\}\right) \\
\leqslant & \quad \int_{\left(m_{1}-\varepsilon\right) \widehat{T} / \mathbb{E} \tau} \bar{F}\left(x+m_{2} t\right) d t+o\left(\mathbb{P}\left\{\zeta_{\widehat{T}}>x+\left(m_{2}-\varepsilon\right) \widehat{T} / \mathbb{E} \tau\right\}\right)
\end{aligned}
$$

for all sufficiently large $\widehat{T}$. Being substituted together with (B.8) into (B.7), it implies that

$$
\psi(x, \widehat{T}, T) \leqslant(1+o(1))\left(\int_{0}^{\mathbb{E} N_{\widehat{T}}} \bar{F}\left(x+m_{1} t\right) d t+\int_{\left(m_{1}-\varepsilon\right) \widehat{T} / \mathbb{E} \tau}^{\mathbb{E} N_{T}} \bar{F}\left(x+m_{2} t\right) d t\right) .
$$

Since $\mathbb{E} N_{\widehat{T}} \sim \widehat{T} / \mathbb{E} \tau$ as $\widehat{T} \rightarrow \infty$ and due to (3.5) with $T=\widehat{T}$, we get

$$
\psi(x, \widehat{T}, T) \leqslant(1+o(1))\left(\int_{0}^{\widehat{T} / \mathbb{E} \tau} \bar{F}\left(x+m_{1} t\right) d t+\int_{\widehat{T} / \mathbb{E} \tau}^{\mathbb{E} N_{T}} \bar{F}\left(x+m_{2} t\right) d t\right)
$$

as $x, T, \widehat{T}, T-\widehat{T} \rightarrow \infty$. So, we obtain

(3.11) $\psi(x, \widehat{T}, T) \leqslant(1+o(1)) \int_{0}^{\mathbb{E} N_{T}} \bar{F}(\widehat{x}(t)) d t \quad$ as $x, T, \widehat{T}, T-\widehat{T} \rightarrow \infty$. 
For the lower bound, let us fix an $\varepsilon>0$ and follow the standard arguments based on the strong law of large numbers and the single big jump principle. By the strong law of large numbers, there exists an $A$ such that

$$
\mathbb{P}\left\{\left|t_{n}-n \mathbb{E} \tau\right|<n \varepsilon+A \text { for all } n \geqslant 1\right\} \geqslant 1-\varepsilon .
$$

On the event introduced in (B.12), if $n \leqslant\left[\frac{\widehat{T}-A}{\mathbb{E} \tau+\varepsilon}\right]=: \widehat{n}$, then $t_{n} \leqslant \widehat{T}$, and hence $n \leqslant N_{\widehat{T}}$. Further, on the event introduced in (B.T2), if $n_{1}:=\left[\frac{\widehat{T}-A}{\mathbb{E} \tau-\varepsilon}\right]<n \leqslant\left[\frac{T-A}{\mathbb{E} \tau+\varepsilon}\right]$ $=: n_{2}$, then $\widehat{T}<t_{n} \leqslant T$, and hence $N_{\widehat{T}}<n \leqslant N_{T}$.

Then, since $\bar{\sigma}$ 's do not depend on the renewal process $N_{t}$, we obtain the inequality

$$
\begin{aligned}
\psi(x, \widehat{T}, T) \geqslant(1-\varepsilon) \mathbb{P}\left\{\sum_{i=1}^{n} \bar{\sigma}_{i}>\widehat{b}(n(\mathbb{E} \tau+\varepsilon)+A)-n \mathbb{E} \sigma\right. \\
\left.\quad \text { for some } n \leqslant \widehat{n} \text { or } n \in\left(n_{1}, n_{2}\right]\right\}
\end{aligned}
$$

Then the standard arguments based on the strong law of large numbers - now for $\sigma$ 's - and the single big jump principle finally imply the lower bound

$$
\psi(x, \widehat{T}, T) \geqslant(1+o(1)) \int_{0}^{\mathbb{E} N_{T}} \bar{F}(\widehat{x}(t)) d t \quad \text { as } x, T, \widehat{T}, T-\widehat{T} \rightarrow \infty,
$$

which together with (B.TI) concludes the proof.

3.3. Proof based on [12]. Let us now give an alternative proof of the asymptotic behaviour of $\psi(x, \widehat{T}, T)$ in the case $T \rightarrow \infty$ based on the results for discrete time from [12].

Let $S_{n}=\sum_{k=1}^{n} \sigma_{k}, \bar{\sigma}_{n}=\sigma_{n}-\mathbb{E} \sigma$, and $\bar{S}_{n}=\sum_{k=1}^{n} \bar{\sigma}_{k} \equiv S_{n}-n \mathbb{E} \sigma$ be a centered random walk. From either Corollary 3 of [13] or Theorem 1 of [9], it follows that, for any random variable $\gamma$ having a light-tailed distribution (that is, $\mathbb{E} \exp (\delta \gamma)<\infty$ for some $\delta>0$ ), we have, for any real $C$,

$$
\mathbb{P}\left\{\bar{S}_{n}>x+n C \text { for some } n \leqslant \gamma\right\} \sim \mathbb{E} \gamma \bar{F}(x) .
$$

Let us take $\varepsilon>0$ sufficiently small, such that $p_{1}(\mathbb{E} \tau-\varepsilon)-\mathbb{E} \sigma>0$ and $p_{2}(\mathbb{E} \tau-\varepsilon)-\mathbb{E} \sigma>0$. We take $\gamma=\min \left\{n \geqslant 1: t_{k} \geqslant(\mathbb{E} \tau-\varepsilon) k\right.$ for all $\left.k \geqslant n\right\}$, which is finite almost surely due to the strong law of large numbers. Moreover, this random variable $\gamma$ is light-tailed because, for $\delta>0$,

$$
\begin{aligned}
\mathbb{P}\{\gamma>n\} & =\mathbb{P}\left\{t_{k}<(\mathbb{E} \tau-\varepsilon) k \text { for some } k \geqslant n\right\} \\
& =\mathbb{P}\left\{e^{\delta\left((\mathbb{E} \tau-\varepsilon) k-t_{k}\right)}>1 \text { for some } k \geqslant n\right\} \leqslant\left(\mathbb{E} e^{\delta(\mathbb{E} \tau-\varepsilon-\tau)}\right)^{n},
\end{aligned}
$$

by Doob's inequality provided that $\mathbb{E} e^{\delta(\mathbb{E} \tau-\varepsilon-\tau)}<1$; it holds for all sufficiently small $\delta>0$ since the random variable $\mathbb{E} \tau-\varepsilon-\tau$ is bounded above and has negative mean. 
Then

$$
\begin{aligned}
& \mathbb{P}\left\{\bar{S}_{n}>\widehat{b}\left(t_{n}\right)-n \mathbb{E} \sigma \text { for some } n \leqslant \gamma\right\} \\
& \qquad \leqslant \mathbb{P}\left\{\bar{S}_{n}>x-n \mathbb{E} \sigma \text { for some } n \leqslant \gamma\right\} \sim \mathbb{E} \gamma \bar{F}(x),
\end{aligned}
$$

by (B.14). Therefore, for all $T>0$,

$$
\begin{aligned}
\psi(x, \widehat{T}, T) \leqslant & \mathbb{P}\left\{\bar{S}_{n}>\widehat{b}\left(t_{n}\right)-n \mathbb{E} \sigma \text { for some } n \leqslant \gamma\right\} \\
& +\mathbb{P}\left\{\bar{S}_{n}>\widehat{b}(n(\mathbb{E} \tau-\varepsilon))-n \mathbb{E} \sigma \text { for some } n \in\left(\gamma, N_{T}\right]\right\} \\
\leqslant & O(\bar{F}(x))+\mathbb{P}\left\{\bar{S}_{n}>\widehat{b}(n(\mathbb{E} \tau-\varepsilon))-n \mathbb{E} \sigma \text { for some } n \leqslant N_{T}\right\}
\end{aligned}
$$

as $x \rightarrow \infty$ uniformly for all $T>0$.

Now we recall the following result where the class $\Gamma$ is a class of all counting random variables $\gamma$ such that, for all $n$, the event $\{\gamma \leqslant n\}$ does not depend on $\left\{\sigma_{k}\right\}_{k>n}$. For $c>0$, let $\mathcal{G}_{c}$ be the class of functions $g$ such that $g(n+1) \geqslant$ $g(n)+c$ for all $n=1,2, \ldots$

THEOREM 3.1 (see [12], Theorem 2(ii)). Assume $\bar{S}_{n}=\sum_{1}^{n} \bar{\sigma}_{n}$ is a centered random walk where the common distribution of $\bar{\sigma}_{n}$ belongs to the class $\mathcal{S}^{*}$. Then, for any $c>0$, uniformly for all $g \in \mathcal{G}_{c}$ and for all random times $\gamma \in \Gamma$, we have

$$
\begin{aligned}
\mathbb{P}\left\{\max _{n \leqslant \gamma}\left(\bar{S}_{n}-g(n)\right)>x\right\} & \sim \sum_{n \geqslant 1} \mathbb{P}(\gamma \geqslant n) \mathbb{P}\left(\bar{\sigma}_{1}>x+g(n)\right) \\
& \sim \sum_{n \geqslant 1} \mathbb{P}(\gamma \geqslant n) \bar{F}(x+g(n)) \quad \text { as } x \rightarrow \infty .
\end{aligned}
$$

Consider a function $g$ of the form $g(n)=\widehat{b}(n(\mathbb{E} \tau-\varepsilon))-n \mathbb{E} \sigma-x$, which is in the class $\mathcal{G}_{p_{1} \wedge p_{2}}$. By Theorem B.], we get

$\mathbb{P}\left\{\bar{S}_{n}>\widehat{b}(n(\mathbb{E} \tau-\varepsilon))-n \mathbb{E} \sigma\right.$ for some $\left.n \leqslant N_{T}\right\} \sim \sum_{n} \mathbb{P}\left\{N_{T} \geqslant n\right\} \bar{F}(x+g(n))$

as $x \rightarrow \infty$ and $T \rightarrow \infty$. Further, since $\varepsilon>0$ is arbitrary, we may follow the proof of the upper bound for $\psi$ and let $\varepsilon \rightarrow 0$ to obtain an upper bound of the form

$$
(1+o(1)) \sum_{n} \mathbb{P}\left(N_{T} \geqslant n\right) \bar{F}(\widehat{x}(n)) .
$$

The last sum may be rewritten as

$$
\begin{aligned}
\mathbb{E} \sum_{n} \mathbb{I}\left(N_{T} \geqslant n\right) \bar{F}(\widehat{x}(n)) & =\mathbb{E} \sum_{n=1}^{N_{T}} \bar{F}(\widehat{x}(n)) \\
& \sim \mathbb{E} \int_{0}^{N_{T}} \bar{F}(\widehat{x}(t)) d t \quad \text { as } x \rightarrow \infty .
\end{aligned}
$$

Here the last equivalence follows from the long-tailedness of $F$. Finally, we may use Lemma 2.1 to conclude with the upper bound (B.T).

Theorem $B .1$ is also applicable to (B.13), therefore the correct lower bound for $\psi(x, \widehat{T}, T)$ follows too. 
3.4. Proof of Lemma 2.1. For any fixed $x$, the function

$$
f(y):=\int_{0}^{y} \bar{F}(x+z(t)) d t
$$

is a concave function in $y$ because $f^{\prime}(y)=\bar{F}(x+z(y))$ is decreasing in $y$. Then the upper bound (2.20) follows by Jensen's inequality for concave functions.

Concerning (2.2I), notice that, for any fixed $\varepsilon>0$,

$$
\mathbb{E} \int_{0}^{N_{T}} \bar{F}(x+z(t)) d t \geqslant \mathbb{P}\left\{N_{T}>(1-\varepsilon) \mathbb{E} N_{T}\right\} \int_{0}^{(1-\varepsilon) \mathbb{E} N_{T}} \bar{F}(x+z(t)) d t,
$$

where $\mathbb{P}\left\{N_{T}>(1-\varepsilon) \mathbb{E} N_{T}\right\} \rightarrow 1$ as $T \rightarrow \infty$ by the law of large numbers for the renewal process, and

$$
\begin{aligned}
\int_{0}^{(1-\varepsilon) \mathbb{E} N_{T}} \bar{F}(x+z(t)) d t & \geqslant(1-\varepsilon) \int_{0}^{\mathbb{E} N_{T}} \bar{F}(x+z((1-\varepsilon) t)) d t \\
& \geqslant(1-\varepsilon) \int_{0}^{\mathbb{E} N_{T}} \bar{F}(x+z(t)) d t
\end{aligned}
$$

implying the lower bound

$$
\mathbb{E} \int_{0}^{N_{T}} \bar{F}(x+z(t)) d t \geqslant(1+o(1)) \int_{0}^{\mathbb{E} N_{T}} \bar{F}(x+z(t)) d t \quad \text { as } x, T \rightarrow \infty,
$$

which together with the upper bound (2.201) justifies (2.2I).

Finally, for any fixed $T$ and $A$, since $z(t) \geqslant 0$ increases, we obtain

$$
\begin{aligned}
& \mathbb{E} \int_{0}^{N_{T}} \bar{F}(x+z(t)) d t \geqslant \mathbb{E}\left\{\int_{0}^{N_{T}} \bar{F}(x+z(t)) d t ; N_{T} \leqslant A\right\} \\
& \geqslant \bar{F}(x+z(A)) \mathbb{E}\left\{N_{T} ; N_{T} \leqslant A\right\} \sim \bar{F}(x) \mathbb{E}\left\{N_{T} ; N_{T} \leqslant A\right\} \quad \text { as } x \rightarrow \infty
\end{aligned}
$$

provided $F$ is long-tailed, in which case also

$$
\int_{0}^{\mathbb{E} N_{T}} \bar{F}(x+z(t)) d t \sim \bar{F}(x) \mathbb{E} N_{T} \quad \text { as } x \rightarrow \infty,
$$

and hence the asymptotics (2.22) follows uniformly on $T$-compact sets. Together with (2.2I) it completes the proof.

3.5. Proof of Theorem 2.2. The proof of this result is very similar to the proof of (2.5) of Theorem 2.1. For example, to prove (2.14) it suffices to observe that

$$
\begin{array}{r}
\mathbb{P}\left\{\frac{\tau_{\wedge}(a x, x)}{e(x)} \geqslant y, \frac{S_{\tau_{\wedge}(a x, x)}-b_{\wedge}(t)}{e(x)} \geqslant v ; \tau_{\wedge}(a x, x)<\infty\right\} \\
=\mathbb{P}\left\{\bar{S}_{n}>\min \left\{a x+p_{1} t_{n}-n \mathbb{E} \sigma, x+p_{2} t_{n}-n \mathbb{E} \sigma\right\}+v e(x)\right. \\
\text { for some } n \geqslant y e(x)\} .
\end{array}
$$


3.6. Generalisation to $n$ insurance companies. It is more or less clear that a very similar proof works for (B.D) if $\widehat{x}(t)$ is a continuous piecewise linear increasing function. Then it allows us to go beyond two insurance companies and consider a model with an arbitrary number of them. Clearly, in high dimension one has to overcome extra combinatorial problems that seem to be doable.

3.7. Open question. We strongly believe that an analogue of Theorem 2.1 holds for stopping times, say $\varrho$ instead of $T$. We expect the following to be correct:

$$
\psi_{\wedge}\left(x_{1}, x_{2}, \varrho\right) \sim \mathbb{E} \int_{0}^{N_{\varrho}} \bar{F}\left(\min \left\{x_{1}+t m_{1}, x_{2}+t m_{2}\right\}\right) d t
$$

and

$$
\psi_{\vee}\left(x_{1}, x_{2}, \varrho\right) \sim \mathbb{E} \int_{0}^{N_{\varrho}} \bar{F}\left(\max \left\{x_{1}+t m_{1}, x_{2}+t m_{2}\right\}\right) d t
$$

hold as $x \rightarrow \infty$ uniformly for all stopping times $\varrho$ with respect to the filtration generated by the renewal process $N_{t}$.

Acknowledgments. We thank Serban Badila for discussions and preliminary results included in his thesis which motivated us to work on the subject and to come to much shorter proofs of more general results given in Theorems $[2$.$] and 2.2$.

\section{REFERENCES}

[1] S. Asmussen and S. Foss, On exceedance times for some processes with dependent increments, J. Appl. Probab. 51 (2014), pp. 136-151.

[2] S. Asmussen and C. Klüppelberg, Large deviations results for subexponential tails, with applications to insurance risk, Stochastic Process. Appl. 64 (1996), pp. 103-125.

[3] F. Avram, Z. Palmowski, and M. Pistorius, Exit problem of a two-dimensional risk process from a cone: Exact and asymptotic results, Ann. Appl. Probab. 18 (2008), pp. 24212449.

[4] F. Avram, Z. Palmowski, and M. Pistorius, A two-dimensional ruin problem on the positive quadrant, Insurance Math. Econom. 42 (2008), pp. 227-234.

[5] S. Badila, O. Boxma, J. Resing, and E. M. M. Winands, Queues and risk models with simultaneous arrivals, Adv. in Appl. Probab. 46 (2014), pp. 812-831.

[6] Y. Chen, Y. Wang, and K. Wang, Asymptotic results for ruin probability of a twodimensional renewal risk model, Stoch. Anal. Appl. 31 (2013), pp. 80-91.

[7] Y. Chen, Y. Wang, and K. Wang, Uniform asymptotics for the finite-time ruin probabilities of two kinds of nonstandard bidimensional risk models, J. Math. Anal. Appl. 401 (2013), pp. 114-129.

[8] Y. Chen, K. Yuen, and K. Ng, Asymptotics for the ruin probabilities of a two-dimensional renewal risk model with heavy-tailed claims, Appl. Stoch. Models Bus. Ind. 27 (3) (2011), pp. 290-300.

[9] D. Denisov, S. Foss, and D. Korshunov, Asymptotics of randomly stopped sums in the presence of heavy tails, Bernoulli 16 (4) (2010), pp. 971-994.

[10] P. Embrechts, C. Klüppelberg, and T. Mikosch, Modelling Extremal Events for Insurance and Finance, Springer, Berlin 1997. 
[11] S. Foss, D. Korshunov, and S. Zachary, An Introduction to Heavy-Tailed and Subexponential Distributions, Springer, 2013.

[12] S. Foss, Z. Palmowski, and S. Zachary, The probability of exceeding a high boundary on a random time interval for a heavy-tailed random walk, Ann. Appl. Probab. 3 (2005), pp. 1936-1957.

[13] S. Foss and S. Zachary, The maximum on a random time interval of a random walk with long-tailed increments and negative drift, Ann. Appl. Probab. 13 (2003), pp. 37-53.

[14] Z. Hu and B. Jiang, On joint ruin probabilities of a two-dimensional risk model with constant interest rate, J. Appl. Probab. 50 (2013), pp. 309-322.

[15] T. Jiang, Y. Wang, Y. Chen, and H. Xu, Uniform asymptotic estimate for finite-time ruin probabilities of a time-dependent bidimensional renewal model, Insurance Math. Econom. 64 (2015), pp. 45-53.

[16] D. G. Konstantinides and J. Li, Asymptotic ruin probabilities for a multidimensional renewal risk model with multivariate regularly varying claims, Insurance Math. Econom. 69 (2016), pp. 38-44.

[17] D. Korshunov, On subexponential tails for the maxima of negatively driven compound renewal and Lévy processes, Stochastic Process. Appl. (to appear); available at https://doi.org/10.1016/j.spa.2017.07.013

[18] J. Li, Z. Liu, and Q. Tang, On the ruin probability of a bidimensional perturbed risk model, Insurance Math. Econom. 41 (2007), pp. 185-195.

[19] P. M. D. Lieshout and M. Mandjes, Tandem Brownian queues, Math. Methods Oper. Res. 66 (2007), pp. 275-298.

[20] D. Lu and B. Zhang, Some asymptotic results of the ruin probabilities in a two-dimensional renewal risk model with some strongly subexponential claims, Statist. Probab. Lett. 114 (2016), pp. 20-29.

[21] T. Rolski, H. Schmidli, V. Schmidt, and J. L. Teugles, Stochastic Processes for Insurance and Finance, Wiley, New York 1999.

[22] Y. Wang, Z. Cui, K. Wang, and X. Ma, Uniform asymptotics of the finite-time ruin probability for all times, J. Math. Anal. Appl. 390 (2012), pp. 208-223.

[23] K. C. Yuen, J. Guo, and X. Wu, On the first time of ruin in the bivariate compound Poisson model, Insurance Math. Econom. 38 (2006), pp. 298-308.

Sergey Foss

Heriot-Watt University, UK

Novosibirsk State University, Russia

Sobolev Institute of Mathematics, Russia

Edinburgh EH14 4AS, Scotland, UK

E-mail:s.foss@hw.ac.uk

Zbigniew Palmowski

Faculty of Pure and Applied Mathematics

Wrocław University of Science and Technology

Wybrzeże Wyspiańskiego 27

50-370 Wrocław, Poland

E-mail: zbigniew.palmowski@gmail.com
Dmitry Korshunov

Lancaster University, UK

Novosibirsk State University, Russia Lancaster, LA1 4YF, United Kingdom

E-mail: d.korshunov@lancaster.ac.uk

Received on 10.2.2017;

revised version on 31.5.2017 
